EXTENDED REPORT

\title{
Effect of laser treatment for dry age related macular degeneration on foveolar choroidal haemodynamics
}

\author{
M Figueroa, L S Schocket, J DuPont, T I Metelitsina, J E Grunwald
}

Br J Ophthalmol 2004;88:792-795. doi: 10.1136/bjo.2003.033837

See end of article for authors' affiliations

.....................

Correspondence to: Dr J E Grunwald,

Department of

Ophthalmology, Scheie

Eye Institute, 51 North

39th Street, Philadelphia,

PA 19104, USA;

juangrun@mail.med.

upenn.edu

Accepted

3 November 2003
Aim: Previous studies have suggested that laser photocoagulation therapy is associated with the resolution of drusen in some age related macular degeneration (AMD) patients. The main aim of the study was to determine whether low intensity laser treatment applied according to the Complications of AMD Prevention Trial (CAPT) protocol produces changes in the choroidal circulation that may help explain the mechanism leading to the resolution of drusen material.

Methods: This ancillary study included 30 CAPT patients with bilateral drusen that were treated and followed at the University of Pennsylvania. Laser Doppler flowmetry was used to measure relative choroidal blood velocity $\left(\mathrm{Ch}_{\text {vel }}\right)$, volume $\left(\mathrm{Ch}_{\text {vol }}\right)$, and flow $\left(\mathrm{Ch}_{\text {flow }}\right)$ in the centre of the fovea. Measurements were obtained through a dilated pupil in both eyes of each patient at the initial CAPT visit before laser treatment was applied in one eye. Measurements were repeated in both eyes of each subject three months later. Analysis of laser Doppler measurements was performed in a masked fashion.

Results: In comparison to baseline, no significant differences in $\mathrm{Ch}_{\text {vel }}, \mathrm{Ch}_{\text {vol }}$, or $\mathrm{Ch}_{\text {flow }}$ were observed three months following the application of low intensity laser according to the CAPT protocol in the untreated and treated eyes. In comparison to the untreated eyes, no significant differences were detected in the treated eyes. Based on the variability of flow measurements in the untreated eyes, the authors estimated an $85 \%$ power to detect a $15 \%$ change in relative blood flow.

Conclusions: The results suggest that large alterations in choroidal blood flow do not occur at three months after low intensity laser therapy following the CAPT protocol.
$\mathrm{T}$ he presence of soft, confluent drusen is a known risk factor for the development of the late complications of age related macular degeneration (AMD). ${ }^{1-5}$ Results of the Choroidal Neovascularisation Prevention Trial (CNVPT) and Prophylactic Treatment of Age-related Macular Degeneration trial have shown that low intensity laser treatment in eyes with drusen is associated with a reduction in the area of drusen..$^{6-8}$ In addition, among the eyes with reduction of drusen in the central macula and no development of choroidal neovascularisation, visual acuity and contrast sensitivity improved by a small amount. ${ }^{6}$ A larger, randomised multicentre trial, the Complications of AgeRelated Macular Degeneration Prevention Trial (CAPT), is now being carried out to evaluate the effects of low intensity laser treatment on the progression of AMD.

We have previously reported evidence suggesting that choroidal blood flow is reduced in the non-exudative stages of AMD. ${ }^{9}$ The choroidal circulation has an important role in the removal of metabolic waste products in the outer retina, and a reduction in blood flow in this vascular tissue could be related to the accumulation of drusen material in AMD.

The purpose of our study is to determine whether low intensity laser treatment applied according to the CAPT protocol alters the dynamics of the foveolar choroidal circulation.

\section{METHODS}

The study population included 30 AMD patients ( 17 female and 13 male) with bilateral drusen who were enrolled in the CAPT at the Scheie Eye Institute. Eligibility was determined according to the CAPT entrance criteria. Mean age was 71 (standard deviation, SD 6) years (range 55 to 82 years). All eyes included in the study had deep anterior chambers and pupillary dilatation greater than $5 \mathrm{~mm}$. All subjects agreed to participate in our study by signing a consent form approved by our institutional review board.

Information about systemic and ocular medications as well as medical and ocular history was obtained from each patient. Eleven patients had a history of hypertension and 10 of them were on systemic therapy. Six patients were on antilipaemic medication. Nineteen were taking multivitamins. One patient had had cataract surgery before the study.

Bilateral pupillary dilatation was achieved with tropicamide $1 \%$ and phenylephrine hydrochloride $2.5 \%$. Measurements of relative foveolar choroidal blood velocity $\left(\mathrm{Ch}_{\mathrm{vel}}\right)$, volume $\left(\mathrm{Ch}_{\mathrm{vol}}\right)$, and flow $\left(\mathrm{Ch}_{\mathrm{flow}}\right)$ were obtained using laser Doppler flowmetry (Oculix instrument) in both eyes of each subject. $\mathrm{Ch}_{\mathrm{vel}}$ represented the speed of moving blood cells whereas $\mathrm{Ch}_{\mathrm{vol}}$ corresponded to the amount of blood present at the measurement site. $\mathrm{Ch}_{\text {flow }}$ was calculated by the instrument from $\mathrm{Ch}_{\mathrm{vel}}$ and $\mathrm{Ch}_{\mathrm{vol}}$. Descriptions of the method have been previously reported.$^{10}$ A $20 \mu \mathrm{W}$ diode laser beam $(670 \mathrm{~nm})$ with a diameter of $200 \mu \mathrm{m}$ was delivered through a fundus camera (Model TRC, Topcon, Tokyo, Japan).

Subjects were asked to fixate on the probing laser beam. A $30^{\circ}$ area of the posterior retina was illuminated at a wavelength of $570 \mu \mathrm{m}$ with a retinal irradiance of approximately $0.03 \mathrm{~mW} / \mathrm{cm}^{2}$ in order to observe the position of the laser beam on the foveola. Proper fixation was confirmed by direct visualisation of the foveola through the fundus camera. This method allows for determinations of choriocapillary flow as discussed by Riva et al. ${ }^{10}$ All measurements were performed with the subjects seated in a darkened room.

Abbreviations: AMD, age related macular degeneration; CAPT, Complications of AMD Prevention Trial; CNVPT, Choroidal Neovascularisation Prevention Trial. 
In both eyes of each patient, three separate 20-30 second measurements of the choroidal circulation were obtained. Following baseline determinations, patients were treated in one eye according to the CAPT protocol. In one eye determined at random, 60 light intensity, $100 \mu \mathrm{m}$ diameter laser photocoagulation burns were applied in a circle at a distance of $1500-2500 \mu \mathrm{m}$ from the centre of the foveola. Three months after treatment, choroidal blood flow determinations were repeated in both eyes.

A trained observer masked to the eye treated, timing of measurements, as well as all other patient's attributes, analysed the data using a NeXT computer (NeXT Computer Inc, Redwood City, CA, USA) with software specifically developed for the analysis of Doppler signals from ocular tissues (NeXT Software Inc).

The masked observer selected segments of the recordings that showed stable circulatory parameters, to avoid unstable blood flow readings produced by disturbances such as eye and head motion, poor fixation, and blinking. Data obtained from the three separate recordings were averaged.

Heart rate, brachial artery systolic $\left(\mathrm{BP}_{\mathrm{s}}\right)$ and diastolic blood pressure $\left(\mathrm{BP}_{\mathrm{d}}\right)$, and intraocular pressure (IOP) measurements were obtained upon completion of laser Doppler flowmetry measurements. Mean blood pressure $\left(\mathrm{BP}_{\mathrm{m}}\right)$ was calculated using the following standard formula:

$$
\mathrm{BP}_{\mathrm{m}}=\mathrm{BP}_{\mathrm{d}}+\mathrm{l} / 3\left(\mathrm{BP}_{\mathrm{s}}-\mathrm{BP}_{\mathrm{d}}\right)
$$

The perfusion pressure (PP) was calculated according to the formula:

$$
\mathrm{PP}=2 / 3 \mathrm{BP}_{\mathrm{m}}-\mathrm{IOP}
$$

Two tailed, paired Student's $t$ tests were employed for the statistical analysis, and p values of less than 0.05 were considered statistically significant.

\section{RESULTS}

No statistically significant changes from baseline were observed in the treated or the untreated eyes at 3 months. In comparison to the untreated eyes, there were also no statistically significant differences in the changes in time in the treated eyes.

Figure 1 shows $\mathrm{Ch}_{\mathrm{vel}}$ measurements obtained at baseline and 3 months in the untreated and treated eyes for each subject. No significant difference in mean $\mathrm{Ch}_{\mathrm{vel}}$ was observed between baseline (0.41 (SD 0.07) arbitrary units (AU); table 1) and 3 month measurements (0.42 (SD 0.09) AU; paired Student's $t$ test, $\mathrm{p}=0.5$ ) in the untreated eyes. Also no significant difference in mean $\mathrm{Ch}_{\mathrm{vel}}$ was observed between baseline (0.42 (SD 0.07) AU; table 2) and 3 month measurements (0.40 (SD 0.07) AU; paired Student's $t$ test, $\mathrm{p}=0.4$ ) in the treated eyes.

Figure 2 shows $\mathrm{Ch}_{\mathrm{vol}}$ measurements at baseline and 3 months in the untreated and treated eyes for each subject. No significant difference in mean $\mathrm{Ch}_{\mathrm{vol}}$ was observed between baseline (0.21 (SD 0.06) AU; table 1) and 3 month measurements (0.21 (SD 0.07) AU; paired Student's $t$ test, $\mathrm{p}=1.0$ ) in the untreated eyes. Also, no significant difference in mean $\mathrm{Ch}_{\mathrm{vol}}$ was observed between baseline (0.19 (SD 0.06) $\mathrm{AU}$; table 2 ) and 3 month measurements (0.20 (SD 0.07) AU; paired Student's $t$ test, $\mathrm{p}=0.4)$ in the treated eyes.

Figure 3 shows for each subject $\mathrm{Ch}_{\text {flow }}$ measurements at baseline and 3 months in the untreated and treated eyes. No significant difference in mean $\mathrm{Ch}_{\text {flow }}$ was observed between baseline (7.3 (SD 2.0) AU; table 1) and 3 months measurements (7.3 (SD 2.5) AU; paired Student's $t$ test, $\mathrm{p}=0.8$ ) in the untreated eyes. Also no significant difference in mean $\mathrm{Ch}_{\text {flow }}$ was observed between baseline (6.8 (SD 1.8) AU;
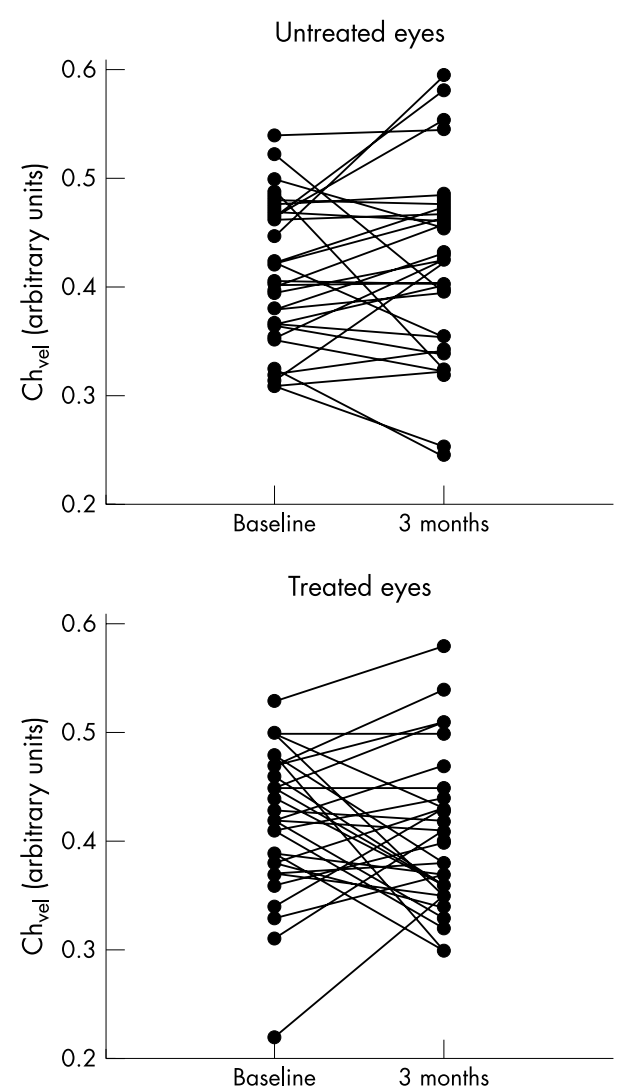

Figure 1 Choroidal blood velocity $\left(\mathrm{Ch}_{\text {vel }}\right)$ in arbitrary units at baseline and 3 months after laser photocoagulation in untreated and treated eyes. There is no significant change in relative $\mathrm{Ch}_{\text {vel }}$ in untreated and treated eyes (paired, two tailed Student's $t$ test; $p=0.5, p=0.4$, respectively).

table 2) and 3 months measurements (7.0 (SD 2.3) AU; paired Student's $t$ test, $\mathrm{p}=0.5$ ) in the treated eyes.

Figure 4 shows for each subject a comparison of the changes in $\mathrm{Ch}_{\text {flow }}$ from baseline observed at 3 months in the untreated and treated eyes. No statistically significant difference was observed between the mean changes in $\mathrm{Ch}_{\text {flow }}$ observed in the untreated and treated eyes (paired Student's $t$ test; $\mathrm{p}=0.6$ ).

There were no significant correlations between blood pressure (systolic, diastolic, and mean), perfusion pressure, or heart rate changes and changes in $\mathrm{Ch}_{\mathrm{vel}}, \mathrm{Ch}_{\mathrm{vol}}$, or $\mathrm{Ch}_{\text {flow }}$.

Based on the variability of flow measurements at three months in the untreated eyes, we estimated that we have an $85 \%$ power to detect a $15 \%$ change in relative blood flow.

\section{DISCUSSION}

A number of studies have suggested that laser photocoagulation treatment can reduce the area of drusen in patients with AMD..$^{6-8}{ }^{11-13}$ Although the exact mechanism by which laser treatment causes drusen resolution is not known, it is

Table 1 Choroidal circulatory parameters in arbitrary units (AU) in untreated eyes. Results shown as mean (SD)

\begin{tabular}{llll}
\hline & Baseline & 3 months & p Value* \\
\hline Ch $_{\text {vel }}$ (AU) & $0.41(0.07)$ & $0.42(0.09)$ & 0.5 \\
h $_{\text {vol }}$ (AU) & $0.21(0.06)$ & $0.21(0.07)$ & 1.0 \\
Ch flow (AU) & $7.3(2.0)$ & $7.3(2.5)$ & 0.8 \\
\hline *Two tailed, paired Student's $t$ test. &
\end{tabular}


Table 2 Choroidal circulatory parameters in arbitrary units $(A U)$ in treated eyes. Results shown as mean (SD)

\begin{tabular}{llll}
\hline & Baseline & 3 months & p Value* \\
\hline Ch $_{\text {vel }}$ (AU) & $0.42(0.07)$ & $0.40(0.07)$ & 0.4 \\
Ch $_{\text {vol }}$ (AU) & $0.19(0.06)$ & $0.20(0.07)$ & 0.4 \\
Chflow (AU) & $6.8(1.8)$ & $7.0(2.3)$ & 0.5 \\
\hline *Two tailed, paired Student's $t$ test. &
\end{tabular}

possible that the choroidal circulation may play a role in this phenomenon. The choroidal circulation is the only vascular supply that nourishes the outer retina. Removal of drusen material and metabolic waste products from the retinal pigment epithelial (RPE) Bruch's complex most probably occurs through this circulatory bed.

Several studies have reported evidence suggesting that choroidal haemodynamic abnormalities are present in AMD. In a prospective study, Holz et $a l^{3}$ found that patients with delayed choroidal perfusion on fluorescein angiography had a higher risk of developing new AMD lesions. Friedman et al ${ }^{14}$ found a significantly higher coefficient of scleral rigidity in AMD patients-a finding that may be associated with increased scleral rigidity that could interfere with venous outflow. Our group has reported, using laser Doppler flowmetry, that choroidal blood flow decreases with age, ${ }^{15}$ and that this decrease is more marked in patients with nonexudative AMD than in age matched controls. ${ }^{9}$ Ciulla et al ${ }^{16}$ have described delayed and heterogeneous filling of the

Untreated eyes
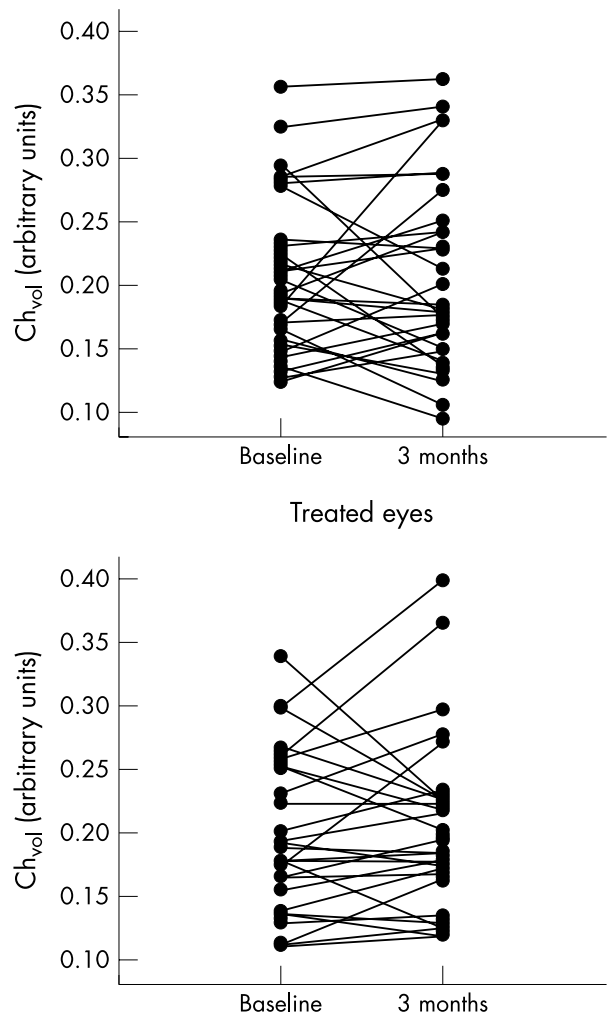

Figure 2 Choroidal blood volume $\left(\mathrm{Ch}_{\text {vol }}\right)$ in arbitrary units at baseline and 3 months after laser photocoagulation in untreated and treated eyes. There is no significant change in relative $\mathrm{Ch}_{\mathrm{vol}}$ in untreated and treated eyes (paired, two tailed Student's $t$ test; $p=1.0, p=0.4$, respectively).
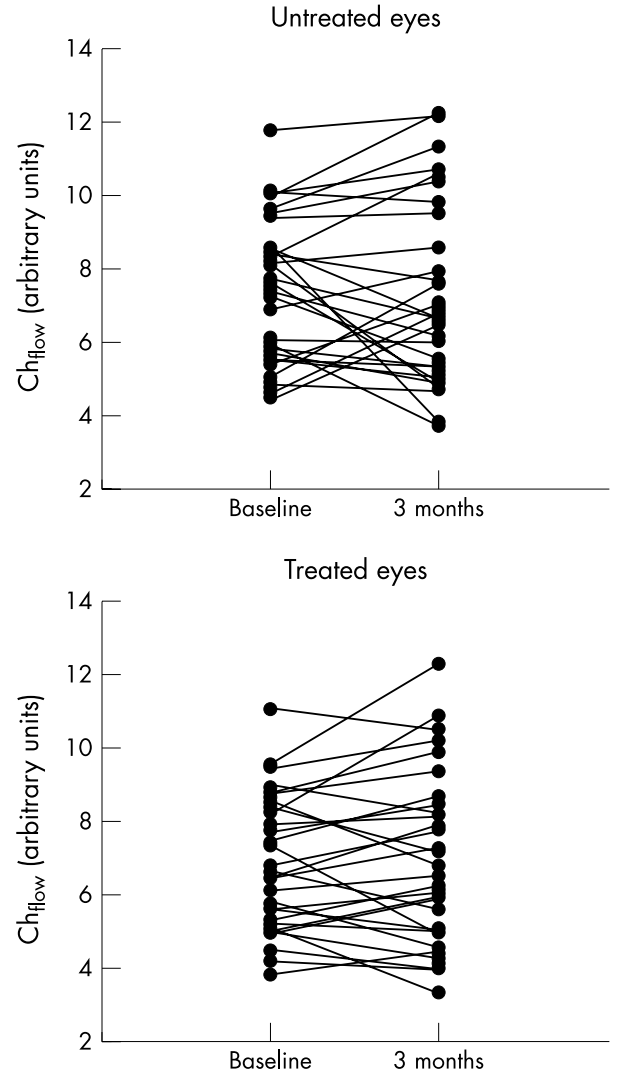

Figure 3 Choroidal blood flow $\left(\mathrm{Ch}_{\text {flow }}\right)$ in arbitrary units $(\mathrm{AU})$ at baseline and 3 months after laser photocoagulation in untreated and treated eyes. There is no significant change in relative $\mathrm{Ch}_{\text {flow }}$ in untreated and treated eyes (paired, two tailed Student's $t$ test; $p=0.8, p=0.5$, respectively).

choroid, particularly in the perifoveal region-a finding that suggests again an abnormality of the choroidal circulation.

Such a reduction in the circulation of the choroid-the only vascular bed that supplies the outer retina-could be related to the accumulation of drusen material in the RPE Bruch's membrane complex. Potentially, an increase in blood flow could lead to a faster removal of metabolic waste products in the RPE Bruch's membrane complex.

To investigate whether choroidal circulatory changes are associated with the reabsorption of drusen material following laser treatment, we have assessed the choroidal circulation just before and 3 months after low intensity laser treatment. Our results, however, do not show any statistically significant

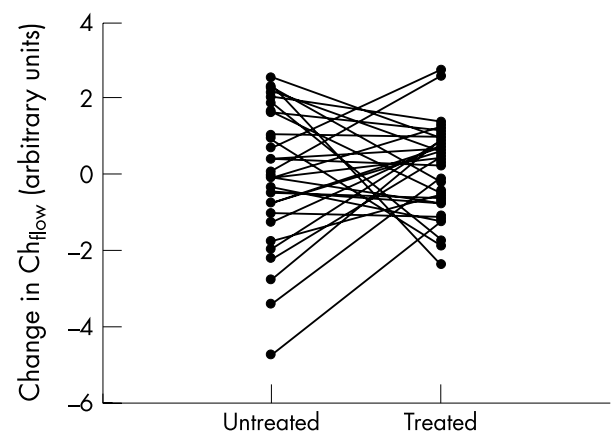

Figure 4 For each subject, a comparison of the change in $\mathrm{Ch}_{\text {flow }}$ in arbitrary units at 3 months between the untreated and the treated eye. No significant difference in $\mathrm{Ch}_{\text {flow }}$ was observed between the untreated and treated eyes (paired, two tailed Student's $t$ test; $p=0.6$ ). 
effect of laser treatment on the choroidal circulation 3 months after treatment. Changes in choroidal blood flow may occur later and we are currently collecting circulatory data two years after the initial low intensity laser treatment to test this hypothesis.

There is evidence to suggest that most of the resolution of drusen following laser treatment may occur later than 3 months. Figueroa et $a l^{11}$ performed a study in which laser was applied directly onto temporal drusen and reported a mean time of drusen disappearance of 2 months. However, the investigators noted that drusen further away from the laser applications resolved more slowly, with a mean time of about 10 months. These results suggest that directly treated drusen resolve at a much faster rate than those present at a distance from the treated site.

The results of the Choroidal Neovascularisation Prevention Trial (CNVPT) support this contention. In this study in which drusen were not treated directly, drusen resolution occurred within 24 months of initial laser photocoagulation treatment, with only relatively few eyes (17\%) showing drusen reduction at 3 months and a larger proportion (43\%) showing this reduction at 6 months. ${ }^{17}$

Because the initial CAPT low intensity laser treatment is not applied directly onto drusen, it is likely that the resolution of drusen takes more than 3 months. As the CAPT is still ongoing, information about the timing and extent of this resolution of drusen material will not be available until the end of the study in 2006.

Another explanation for the lack of significant change in circulatory parameters at three months may be that in our study we are assessing the choroidal circulation in the centre of the fovea, an area that is not directly treated with laser spots in the CAPT protocol.

Because we did not find any significant effect of laser treatment on the choroidal circulation at 3 months after treatment, we have calculated the statistical power that we have to detect circulatory changes. Based on the variability of flow measurements at 3 months in the untreated eyes, we estimated that we have an $85 \%$ power to detect a $15 \%$ change in relative blood flow.

We are currently investigating the changes that occur in the choroidal circulation 2 years after low intensity laser treatment to assess whether such changes may occur after 3 months. Because the CAPT study is being carried out in a masked fashion, we do not have data on which specific eyes have shown resolution of drusen. When this information is released in 2006, we will be able to test whether patients who show resolution of drusen after treatment have choroidal circulatory changes that are different from those eyes that do not show resolution.

\section{Authors' affiliations}

M Figueroa, L S Schocket, J DuPont, T I Metelitsina, J E Grunwald, Department of Ophthalmology, Scheie Eye Institute, School of Medicine, University of Pennsylvania, Philadelphia, PA, USA

\section{REFERENCES}

1 Smiddy WE, Fine SL. Prognosis of patients with bilateral macular drusen. Ophthalmology 1984;91:271-7.

2 Bressler SB, Maguire MG, Bressler NM, et al. Relationship of drusen and abnormalities of the retinal pigment epithelium to the prognosis of neovascular macular degeneration. The Macular Photocoagulation Study Group. Arch Ophthalmol 1990; 108:1442-7.

3 Holz FG, Wolfensberger TJ, Piguet B, et al. Bilateral macular drusen in agerelated macular degeneration. Ophthalmology 1994;101:1522-8.

4 Klein R, Klein BEK, Jensen SC, et al. The five-year incidence and progression of age-related maculopathy: the Beaver Dam Eye Study. Ophthalmology 1997; 104:7-21.

5 Wang JJ, Foran S, Smith W, et al. Risk of age-related macular degeneration in eyes with macular drusen or hyperpigmentation. The Blue Mountains Eye Study Cohort. Arch Ophthalmol 2003;121:658-63.

6 Ho AC, Maguire MG, Yoken J, et al. Laser-induced drusen reduction improves visual function at 1 year. Ophthalmology 1999;106:1367-74.

7 Olk RJ, Friberg TR, Stickney KL, et al. Therapeutic benefits of infrared (810$\mathrm{nm}$ ) diode laser macular grid photocoagulation in prophylactic treatment of nonexudative age-related macular degeneration: two-year results of a randomized pilot study. Ophthalmology 1999;106:2082-90.

8 Rodanant N, Friberg TR, Cheng L, et al. Predictors of drusen reduction after subthreshold infrared $(810 \mathrm{~nm})$ diode laser macular grid photocoagulation for nonexudative age-related macular degeneration. Am J Ophthalmol 2002; 134:577-85.

9 Grunwald JE, Hariprasad SM, DuPont J, et al. Foveolar choroidal blood flow in age-related macular degeneration. Invest Ophthalmol Vis Sci 1998;39:385-90.

10 Riva CE, Cranstoun SD, Grunwald JE, et al. Choroidal blood flow in the foveal region of the human ocular fundus. Invest Ophthalmol Vis Sci 1994;35:4273-81.

11 Figueroa MS, Regueras A, Bertrand J. Laser photocoagulation to treat macular soft drusen in age-related macular degeneration. Retina 1994; 14:391-6.

12 Little HL, Showman JM, Brown BW. A pilot randomized controlled study on the effect of laser photocoagulation of confluent soft macular drusen. Ophthalmology 1997; 104:623-31.

13 Frennesson C, Nilsson SEG. Prophylactic laser treatment in early related maculopathy reduced the incidence of exudative complications. Br J Ophthalmol 1998:1169-74.

14 Friedman $E$, Ivry $M$, Ebert $E$, et al. Increased scleral rigidity and age-related macular degeneration. Ophthalmology 1989;96:104-8.

15 Grunwald JE, Hariprasad SM, DuPont J. Effect of aging on foveolar choroidal circulation. Arch Ophthalmol 1998;116:150-4.

16 Ciulla TA, Harris A, Kagemann L, et al. Choroidal perfusion perturbations in non-neovascular age related macular degeneration. $\mathrm{Br} J$ Ophthalmol 2002;86:209-13.

17 The Choroidal Neovascular Prevention Trial Research Group. Laser treatment in fellow eyes with large drusen: updated findings from a pilot randomized clinical trial. Ophthalmology 2003;110:971-8. 